Check for updates

Cite this: RSC Adv., 2018, 8, 38556

Received 29th September 2018 Accepted 1st November 2018

DOI: $10.1039 / \mathrm{c} 8 \mathrm{ra} 08073 a$

rsc.li/rsc-advances

\section{Experimental verification of asymmetric transmission in continuous omega-shaped metamaterials}

\author{
Ying-Hua Wang, (D) $\dagger^{\mathrm{ab}}$ Inki Kim, $\dagger^{\mathrm{c}}$ Ren-Chao Jin, ${ }^{\mathrm{b}}$ Heonyeong Jeong, ${ }^{\mathrm{c}}$ Jia-Qi Li, ${ }^{\mathrm{b}}$ \\ Zheng-Gao Dong ${ }^{\star b}$ and Junsuk Rho (D) *cd
}

\begin{abstract}
A bi-layer continuous omega-shaped metamaterial was proposed and fabricated to measure the asymmetric transmission (AT) effect of a linearly polarized light at near-infrared region. The metamaterial was fabricated by the electron-beam lithography method, and the AT effect was demonstrated by the difference between total transmittances in the two opposite propagation directions for $x$ - $/ y$-polarized incident light. The experimental results were confirmed by the full-wave simulated results. Importantly, we also experimentally demonstrated that the AT effect is robust against the misalignments between the first and the second omega-shaped layers. Accordingly, the successfully prepared sample and its characterization provide a bright future for applications in light-controlled switchers and optical diodes in on-chip optical systems and information communication systems.
\end{abstract}

\section{Introduction}

Metamaterials are artificial media with great scientific and application values as they can arbitrarily manipulate the electromagnetic waves from microwave to optical region by adjusting their shapes, sizes and arrangements. For instance, asymmetric transmission (AT) effects, optical activities, perfect absorptions and other electromagnetic effects have already been realized by I-shaped resonators, ${ }^{1,2}$ helix photonic metamaterials, ${ }^{3-5}$ fish-scale metallic wires,${ }^{6}$ cross-shaped resonators ${ }^{7}$ and so on. In addition, "digital metamaterials" have also been proposed, though which the electromagnetic waves can be manipulated more flexibly by coding ' 0 ' and ' 1 ' elements with controlled sequences (i.e., 1 bit coding). ${ }^{8}$ Recently, metamaterials have been studied widely by simulation methods; most of the sample fabrications and experimental measurements were just realized at microwave and terahertz regions by both two-dimensional (2D) and three-dimensional (3D) metamaterials. ${ }^{1,2,9}$ In contrast, the fabrication of nanoscale metastructures at infrared and optical regions primarily involve planar metasurfaces rather than $3 \mathrm{D}$ metamaterials. ${ }^{7, \mathbf{1 0}, 11}$

\footnotetext{
${ }^{a}$ Shandong Provincial Key Laboratory of Laser Polarization and Information Technology, Laser Institute, Qufu Normal University, Qufu 273165, China. E-mail: wyh121@qfnu.edu.cn

${ }^{b}$ School of Physics, Southeast University, Nanjing 211189, China. E-mail: zgdong@seu. edu.cn

${ }^{c}$ Department of Mechanical Engineering, Pohang University of Science and Technology (POSTECH), Pohang 37673, Republic of Korea

${ }^{d}$ Department of Chemical Engineering, Pohang University of Science and Technology (POSTECH), Pohang 37673, Republic of Korea. E-mail: jsrho@postech.ac.kr

$\dagger$ These authors contributed equally to the paper.
}

However, it is of great significance to overcome the challenges of fabricating 3D metamaterials at nanoscale, which have presented or enhanced a variety of electromagnetic properties not realized in planar metamaterials, such as AT effect of linearly polarized light ${ }^{\mathbf{1 2 , 1 3}}$ and the enhanced optical activity. ${ }^{\mathbf{1 4 , 1 5}}$

The AT effect indicates that the total transmittance (transmitted intensity divided by the incident intensity) is different when incident light propagates in opposite directions. ${ }^{\mathbf{1 6}}$ Over the past decade, asymmetric transmission has attracted great attention and has presented potential applications in optical isolators, switchers and other electromagnetic devices. For flexibly and dynamically controlling the asymmetric electromagnetic waves, PIN diodes were provided by putting the external stimulation of voltage biasing to the bilayer twisted split-ring resonators. ${ }^{17}$ Moreover, many other studies introduced graphene and disturbance to adjust the asymmetric transmissions. ${ }^{18-20}$ Traditionally, magneto-optical materials ${ }^{\mathbf{2 1 , 2 2}}$ and non-linear media ${ }^{2324}$ were used to break the Lorentz's symmetry condition and induce the effect of nonreciprocal transmissions. However, these studies usually involve a material whose size is bigger than the desired wavelength; hence, they cannot be used in the micro-nano optical system and other optical communication systems. In addition, the gradient metasurface, ${ }^{25}$ asymmetric gratings,,${ }^{\mathbf{9} 26,27}$ and chiral metamaterials ${ }^{28,29}$ have been proposed to realize AT effects without breaking Lorentz's reciprocity theorem. It is complicated to properly design the phase gradient of the metasurface ${ }^{25}$ or to avoid the higher diffraction modes of asymmetric gratings. ${ }^{26}$ However, it is various and flexible in desiging 2D and 3D chiral metamaterials. Moreover, the transmissions for the chiral metamaterials are either co-polarized or cross-polarized 
without higher diffraction modes. Therefore, many subwavelength-scale chiral metamaterials are proposed to realize the circularly and linearly polarized AT effects. ${ }^{\mathbf{1 , 1 6 , 3 0 , 3 1}}$ It is well known that 2D metamaterials cannot destroy mirror symmetry in the propagation direction normal to the $2 \mathrm{D}$ plane, so only circularly polarized AT effect can be realized by $2 \mathrm{D}$ metamaterials. ${ }^{10,12,32}$ However, the $3 \mathrm{D}$ metamaterials without mirror symmetries in the directions parallel and perpendicular to the propagation of light can induce circularly or linearly polarized AT effects. ${ }^{28,33}$ For example, 3D metamaterials without any rotational symmetry have been proposed to realize the circularly and linearly polarized AT effects, concurrently. ${ }^{11,34}$

In this study, we proposed the well-aligned bi-layer continuous omega-shaped metamaterials and experimentally demonstrated the AT effect for linearly polarized incident light. Compared with the previously demonstrated wavelength range, ${ }^{35}$ it is significant that the operating wavelength range band is brought to a shorter near-infrared wavelength range, which is highly demanded for practical optical applications. To show the AT phenomena clearly and intuitively, we measured and simulated total transmittances for $x$ - and $y$-polarized incident lights propagating along the forward and backward directions. Then, we confirmed that the experimental results were in good agreement with the full-wave simulations. More importantly, we experimentally verified that the AT effect is robust to the misaligned errors (particularly lateral errors, not rotational errors) of this bi-layer continuous omega-shaped metamaterial. The successful experiment and robustness of the design will provide potential applications for on-chip optical computing, optical diode and other optical communication systems.

\section{Sample and design}

Fig. 1(a) presents the scanning electron microscopy (SEM) image of the bi-layer continuous gold $(\mathrm{Au})$ omega-shaped metamaterial embedded in a silica substrate, and the whole coverage area of the sample is $50 \mu \mathrm{m} \times 50 \mu \mathrm{m}$. Schematics of the unit cell for perspective view and top view are presented in Fig. 1(b) and (c), respectively, with dimensions of the fabricated structure as $a=330 \mathrm{~nm}, b=330 \mathrm{~nm}, w=80 \mathrm{~nm}, t=40 \mathrm{~nm}$ and $L=500 \mathrm{~nm}$. It is worth mentioning that the distance between the top and bottom layer was $d=120 \mathrm{~nm}$, which was the minimum distance for our fabrication. In order to demonstrate our experiment result, we have performed Finite Difference Time Domain (FDTD) method. And gold is used by a Drude-type model with plasma frequency $\omega_{\mathrm{p}}=1.367 \times 10^{16} \mathrm{rad} \mathrm{s}^{-1}$ and collision frequency $\gamma=4.0715 \times 10^{13} \mathrm{rad} \mathrm{s}^{-1} \cdot{ }^{35}$ Meanwhile, a silicon-oxide with $\varepsilon_{\mathrm{SiO}_{2}}=2.1$ is considered as dielectric environment. Herein, we used periodic boundary conditions in $x$ and $y$-directions to realize the continuous omega-shaped metamaterial, and the total transmittances were probed directly when the $x$ - and $y$-polarized incident lights propagated along the forward $(+z)$ and backward $(-z)$ directions.

For sample fabrication, the polymethyl methacrylate (PMMA) layer was first spin-coated onto the $500 \mu \mathrm{m}$-thick silica $\left(\mathrm{SiO}_{2}\right)$ substrate. Similarly, a conductive polymer layer (Showa

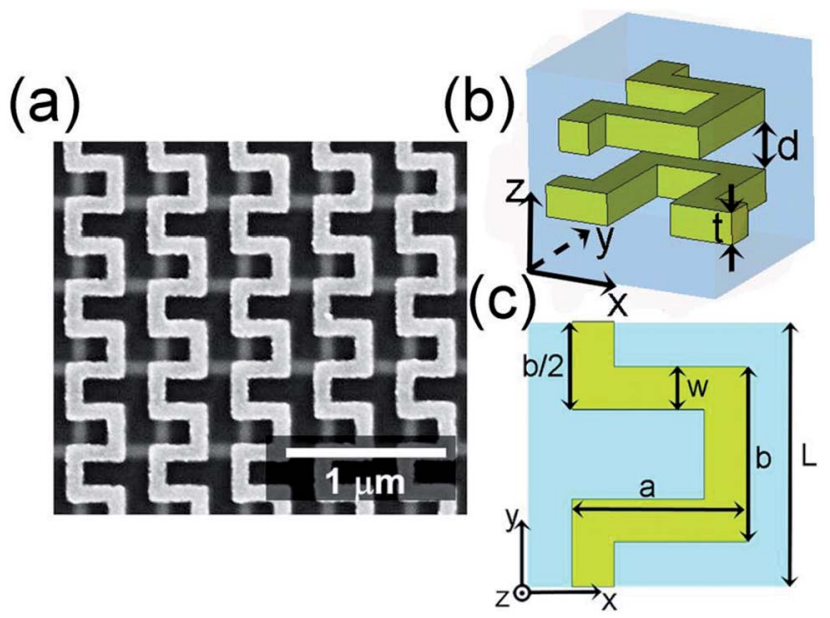

Fig. 1 (a) SEM image of the fabricated omega-shaped metamaterial. (b) Perspective view of the unit cell with bi-layer continuous omegashaped Au metamaterial embedded in $\mathrm{SiO}_{2}$. (c) Top view of the upper continuous omega-shaped metamaterial.

Denko, E-spacer 300Z) was also spin-coated to prevent the charging effect from the dielectric substrate. Then, the continuous omega-shaped pattern was exposed by an electron-beam lithography (EBL) system (ELIONIX ELS-7800, $80 \mathrm{kV}, 50 \mathrm{pA}$ ). After exposing, the conductive polymer layer was first removed by DI water and then, the continuous omega-shaped pattern was developed and fixated by the MIBK : IPA $1: 3$ solutions and IPA. Then, an adhesion layer with $3 \mathrm{~nm}$-thick chrome and a structure layer with $40 \mathrm{~nm}$-thick $\mathrm{Au}$ were deposited by electron-beam evaporation (KVT KVE-ENS4004). Hence, the first (bottom) continuous omega-shaped Au layer was transferred onto the substrate by a standard lift-off process. Next, on top of the first layer, a $120 \mathrm{~nm}$-thick spin-on-glass polymer layer was spin-coated and baked. The lithography processes was repeated along with an ultra-accurate overlay process ${ }^{36}$ to obtain the second (upper) continuous omega-shaped Au layer. Finally, another $\mathrm{SiO}_{2}$ layer was deposited on top of the sample to encapsulate the continuous omega-shaped metamaterial in $\mathrm{SiO}_{2}$ environment.

\section{Experimental results}

It is known that the AT parameters $(\Delta)$ for linearly polarized light can be calculated by $\Delta_{x}=t_{x}^{\mathrm{f}}-t_{x}^{\mathrm{b}}$ and $\Delta_{y}=t_{y}^{\mathrm{f}}-t_{y}^{\mathrm{b}},{ }^{37}$ where $\mathrm{f}$ and $\mathrm{b}$ indicate that the incident lights propagate along the forward and backward directions, respectively, and $t_{x}$ and $t_{y}$ indicate the total transmittances for the $x$ - and $y$-polarized incident lights, respectively. Fig. 2 presents a homemade optical setup for measuring the spectra of total transmittances for both $x$ - and $y$-polarized incident lights. In this experiment, we rotated the sample $180^{\circ}$ along the $x$-direction for realizing the forward and backward illuminations. The white light (Ocean Optics, HL 2000) was first calibrated by the convex lens (L1). The convex lens from L2 to L4 ensured parallel illumination, and $x$ - and $y$ polarized incident lights could be induced by the linear polarizer (P). In addition, diaphragms (D1, D2) and a filter (F) were 


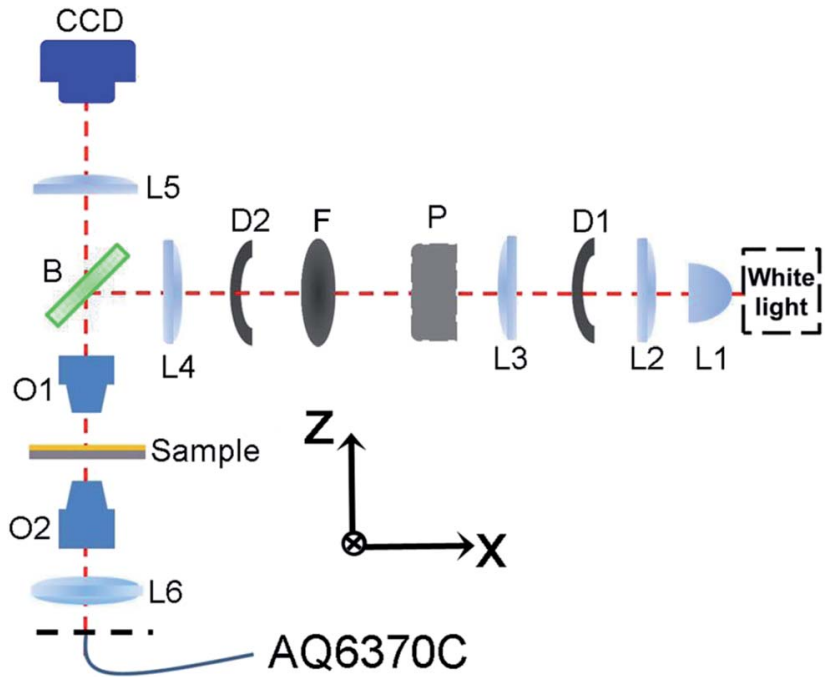

Fig. 2 Schematic of the optical path designed for measuring the total transmittances for the $x$ - and $y$-polarized incident light.

used to control the faculae and light intensity. Then, the illuminated light was reflected to the objective 1 (O1: Olympus, $50 \times$, N.A. 0.8 ) by the beam splitter (B) and focused on the sample. As a result, the light separated into two paths after interacting with the continuous omega-shaped metamaterial: one path light was focused on the charge-coupled device (CCD) to observe the sample, and the other path light passed through the sample and was collected by the objective $2(\mathrm{O} 2$ : Olympus, $50 \times$, N.A. 0.5$)$ and measured by the spectroscopy analyzer (AQ6370C).

\section{Results and discussion}

In order to clearly show the differences in total transmittances for linearly polarized light propagating along the two opposite directions, we have measured the total transmittances directly rather than the transmission coefficients in Jones matrix in our previous studies. ${ }^{35,38}$ Fig. 3(a) and (b) present the measured spectra of total transmittances recorded by the homemade optical setup shown in Fig. 2. For $x$-polarized incident light [Fig. 3(a)], the total transmittance for backward $(-z)$ propagation $\left(t_{x}^{\mathrm{b}}\right)$ is about zero at around $1350 \mathrm{~nm}$. In addition, the transmittance in forward $\left(t_{x}^{\mathrm{f}}\right)$ direction is 0.12 at around $1350 \mathrm{~nm}$, which can be considered as an efficient transmittance compared with previous reports. ${ }^{29,39}$ Therefore, $x$-polarized incident light could pass through the continuous omegashaped metamaterial along the forward direction, but it was prevented along the backward direction. As discussed in the former simulation study, it is known that the hybrid resonance and chiral arrangement of the bi-layer metamaterial result in a high transmittance for $x$-polarized incident light propagating along the forward direction. ${ }^{37}$ Moreover, the bi-layer metamaterial cannot induce a hybrid resonance for the $x$-polarized incident light propagating along the backward direction. Therefore, the backward transmittance is nearly zero. For the $y$ polarized incident light [Fig. 3(b)], only backward direction is
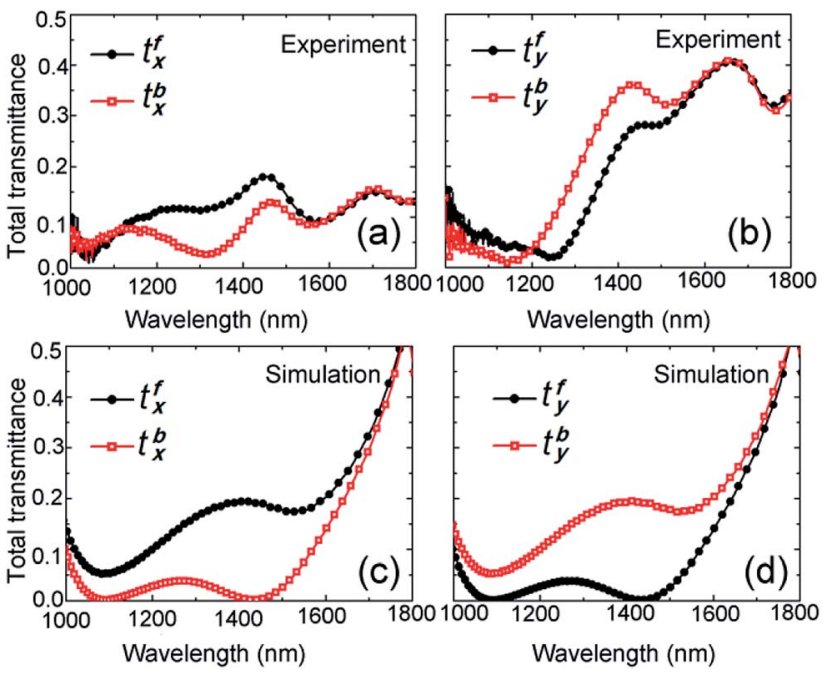

Fig. 3 Measured (a) and (b) and simulated (c) and (d) total transmittances for $x$ - and $y$-polarized incident light propagating along the forward and backward directions.

allowed for the light to propagate through the sample rather than the forward direction. To confirm the experimental results, we performed simulations to numerically check the asymmetric total transmittances for the $x$ - and $y$-polarized incident lights propagating along the forward and backward directions, as shown in Fig. 3(c) and (d). The simulated results show that the $x$-polarized incident light can only pass through the continuous omega-shaped metamaterial along the forward direction at a resonance wavelength of about $1400 \mathrm{~nm}$. Inversely, the $y$ polarized incident light can only pass through the metamaterial along the backward direction at the same resonant wavelength. The overall tendency of the simulated spectra is in reasonable agreement with the measured result. Herein, the simulated total transmittances for $x$ - and $y$-polarized incident lights propagating along the forward and backward directions were consistent $\left(t_{x}^{\mathrm{f}}=t_{y}^{\mathrm{b}}\right.$ and $\left.t_{x}^{\mathrm{b}}=t_{y}^{\mathrm{f}}\right)$, which could be attributed to the spatial symmetry of the proposed bi-layer metamaterial. However, for the experiment, it is hard to keep an exactly $x$ - $/ y$ polarized incident light; as a result, the spatial symmetry is destroyed and the consistency disappeared. In addition, the fabrication errors in critical dimensions of the structures, uniformity coefficient of the middle coating film, noises of our homemade optical setup and other disturbances were responsible for the differences between experimental data in Fig. 3(a) and (b) and simulations in Fig. 3(c) and (d).

Fig. 4(a) and (b) show the AT parameters for the experiment and simulation calculated by the equation $\Delta_{x}=t_{x}^{\mathrm{f}}-t_{x}^{\mathrm{b}}=-\left(t_{y}^{\mathrm{f}}-\right.$ $\left.t_{y}^{\mathrm{b}}\right)=-\Delta_{y}$. It is can be clearly found that the maximum values of AT peaks are about \pm 0.1 for the experiment, which agrees well with the simulated result of about \pm 0.18 . The AT peaks appeared at around $1350 \mathrm{~nm}$ in the measured results, which suffered a slight blue shift compared with the simulations. As discussed in previous studies, the blue shift should come from the fabrication errors for parameter $a$ in Fig. 1(c). ${ }^{37}$ Fig. 4(c) presents the AT efficiencies of the simulated (Sim.) and experimental (Exp.) results, which are shown by dotted and solid 

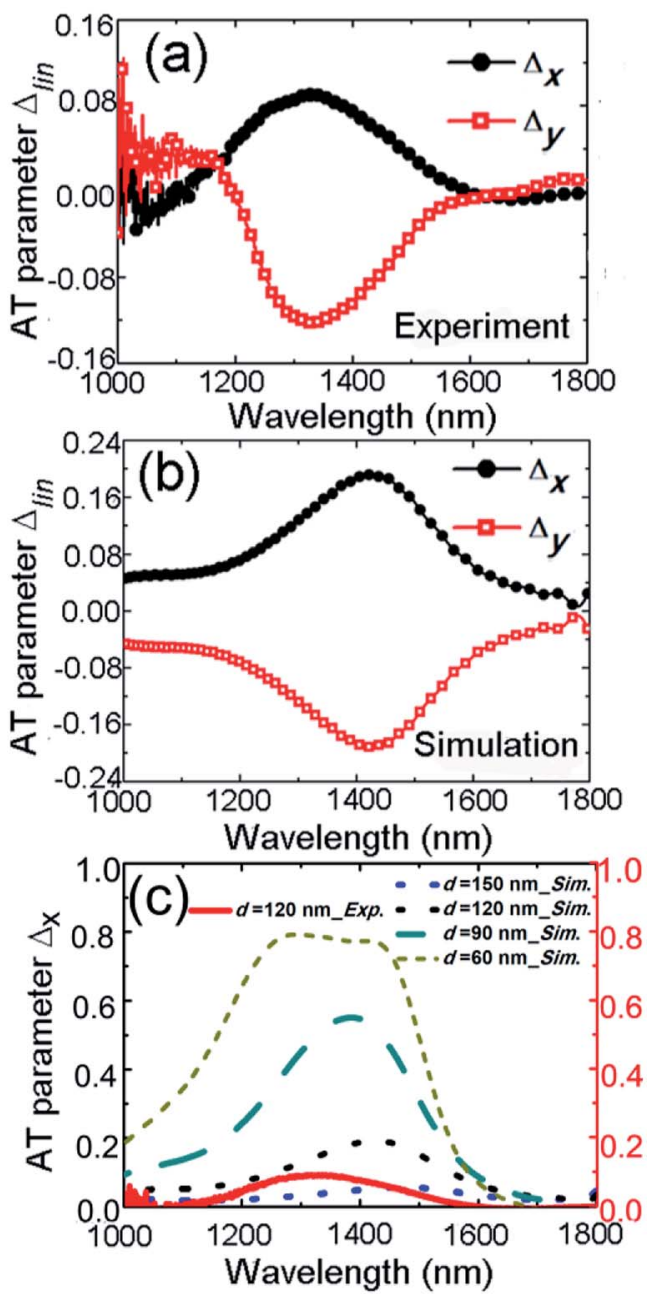

Fig. 4 Measured (a) and simulated (b) asymmetric transmissions for $x$ and $y$-polarized lights; (c) asymmetric transmissions influenced by distance $d$ between top and bottom layers. lines, respectively. From the simulated results, we can infer that for the continuous omega-shaped metamaterials, the AT parameters are sensitive to distance $(d)$ between top and bottom layers, and the AT parameter reached up to 0.8 at $d=60 \mathrm{~nm}$. However, the AT parameter is only 0.05 at $d=150 \mathrm{~nm}$, which could be attributed to the resonance of Fabry-Perot-like cavity; also, the interactions between the incident wave and the bi-layer omega-shaped metamaterial are all weak. ${ }^{37,40}$ In addition, it was necessary to realize the AT effect experimentally,. Hence, in this study, we paid more attention on sample fabrications and AT measurements. However, it is still a challenge to decrease the distance to $d=60 \mathrm{~nm}$. This is because if the thickness of the spacing layer is too low, the first layer cannot be uniformly covered and planarization cannot be achieved. Hence, the distance $d$ could be decreased down to only $120 \mathrm{~nm}$ using spinon-glass in this experimental study; as a result, the AT parameter could just reach nearly 0.1. ${ }^{37}$ Therefore, it is of great importance to further overcome the challenges in fabrications to realize better AT effects experimentally. Moreover, the multilayered programmable metasurfaces have been proposed to improve the transmission at microwave and $\mathrm{THz}$ frequencies. ${ }^{\mathbf{4 1 , 4 2}}$ Hence, increasing the layers of our omega-structure would be also considered to improve the AT efficiency at optical frequencies in future.

To study the influence of overlay fabrication imperfections, we experimentally examined the AT effect of the misaligned continuous omega-shaped metamaterial. Fig. 5(a) presents the SEM image of a misaligned omega-shaped sample with misalignments $\delta_{x}=135 \mathrm{~nm}$ and $\delta_{y}=50 \mathrm{~nm}$. Then, total transmittances were measured by the same homemade optical setup. As presented in Fig. 5(b) and (c), the tendency of transmittance spectra is consistent with the spectra obtained from the original well-aligned sample in Fig. 2. Then, the corresponding AT parameters were calculated and shown in Fig. 5(d)

\section{(a)}
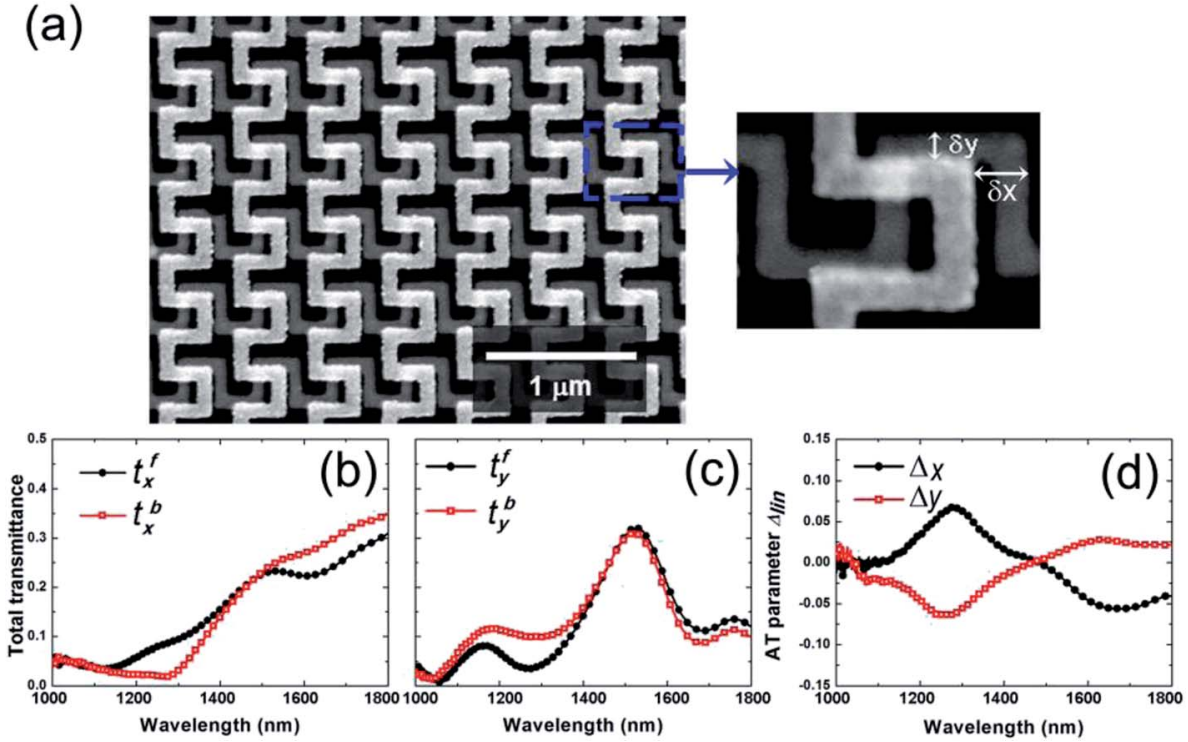

Fig. 5 (a) SEM image of the fabricated continuous omega-shaped metamaterial with lateral errors in $x$ - and $y$-directions. (b) and (c) Measured total transmittances for $x$ - and $y$-polarized incident lights propagating along forward and backward directions, respectively. (d) The corresponding asymmetric transmissions. 
with efficient AT values of about $\pm 0.075 .{ }^{29}$ The working wavelength range and values of AT parameters were also approximated to the result in Fig. 4(a), where slight deviations could be attributed to uncontrollable factors in fabrications and measurements. Finally, we can conclude that the AT effect is robust towards the lateral misalignments between the top and bottom omega-shaped layers.

\section{Summary}

In summary, we have experimentally demonstrated the AT effect for linearly polarized light by continuous omega-shaped metamaterial, and the measured results were also confirmed by FDTD simulations. For fabrications, the alignment errors caused by the EBL machine were minimized to obtain wellaligned samples. In addition, we propose a simple homemade optical setup, which can measure the total transmittances of $x$-/ $y$-polarized incident lights. Then, the forward and backward total transmittances can be realized by rotating the sample, so that the AT effect can be measured experimentally. Finally, we have also experimentally verified that our continuous omegashaped metamaterial is robust against misalignment errors, which are unavoidable in fabrications, showing its high possibility in further practical applications.

\section{Conflicts of interest}

There are no conflict to declare.

\section{Acknowledgements}

This study was supported by the National Natural Science Foundation of China (No. 11774053, 11374049, 11511140278); National Research Foundation of Korea (NRF2017R1E1A1A03070501, NRF-2015R1A5A1037668, CAMM2014M3A6B3063708, NRF-2017R1E1A2A01076613); Ministry of Science and ICT of the Korean government (NRF2016H1A2A1906519).

\section{Notes and references}

1 J. Shi, X. Liu, S. Yu, T. Lv, Z. Zhu, H. Feng Ma and T. Jun Cui, Appl. Phys. Lett., 2013, 102, 191905.

2 S. Fang, K. Luan, H. F. Ma, W. Lv, Y. Li, Z. Zhu, C. Guan, J. Shi and T. J. Cui, J. Appl. Phys., 2017, 121, 033103.

3 Y. R. Li and Y. C. Hung, Opt. Express, 2015, 23, 16772-16781. 4 C. Wu, H. Li, X. Yu, F. Li, H. Chen and C. T. Chan, Phys. Rev. Lett., 2011, 107, 177401.

5 J. K. Gansel, M. Thiel, M. S. Rill, M. Decker, K. Bade, V. Saile, G. von Freymann, S. Linden and M. Wegener, Science, 2009, 325, 1513-1515.

6 J. Han, H. Li, Y. Fan, Z. Wei, C. Wu, Y. Cao, X. Yu, F. Li and Z. Wang, Appl. Phys. Lett., 2011, 98, 151908.

7 W. Ma, Y. Wen and X. Yu, Opt. Express, 2013, 21, 3072430730.

8 T. J. Cui, M. Q. Qi, X. Wan, J. Zhao and Q. Cheng, Light: Sci. Appl., 2014, 3, e218.
9 D. Yavorskiy, M. Stolarek, J. Lusakowski and R. Kotynski, Opt. Express, 2014, 22, 30547-30552.

10 A. S. Schwanecke, V. A. Fedotov, V. V. Khardikov, S. L. Prosvirnin, Y. Chen and N. I. Zheludev, Nano Lett., 2008, 8, 2940-2943.

11 M. Kang, H. T. Wang and W. Zhu, Opt. Express, 2014, 22, 9301-9306.

12 Z. Li, M. Mutlu and E. Ozbay, J. Opt., 2013, 15, 023001.

13 Z. Wang, F. Cheng, T. Winsor and Y. Liu, Nanotechnology, 2016, 27, 412001.

14 Y. Zhao, M. A. Belkin and A. Alu, Nat. Commun., 2012, 3, 870. 15 R. Ji, S. W. Wang, X. Liu and W. Lu, Nanoscale, 2016, 8, 81898194.

16 G. Kenanakis, A. Xomalis, A. Selimis, M. Vamvakaki, M. Farsari, M. Kafesaki, C. M. Soukoulis and E. N. Economou, ACS Photonics, 2015, 2, 287-294.

17 K. Chen, Y. Feng, L. Cui, J. Zhao, T. Jiang and B. Zhu, Sci. Rep., 2017, 7, 42802.

18 Z. Li, W. Liu, H. Cheng, S. Chen and J. Tian, Opt. Lett., 2016, 41, 3142-3145.

19 H. Jiang, W. Zhao and Y. Jiang, Opt. Express, 2017, 25, 1973219769.

20 J. Zhao, Y. Fu, Z. Liu and J. Zhou, Opt. Express, 2017, 25, 23051-23059.

21 C. He, X.-L. Chen, M.-H. Lu, X.-F. Li, W.-W. Wan, X.-S. Qian, R.-C. Yin and Y.-F. Chen, Appl. Phys. Lett., 2010, 96, 111111. 22 H. Zhu and C. Jiang, Opt. Lett., 2011, 36, 1308-1310.

23 N. Bender, S. Factor, J. D. Bodyfelt, H. Ramezani, D. N. Christodoulides, F. M. Ellis and T. Kottos, Phys. Rev. Lett., 2013, 110, 234101.

24 S. Lepri and G. Casati, Phys. Rev. Lett., 2011, 106, 164101.

25 Y. Ling, L. Huang, W. Hong, T. Liu, Y. Sun, J. Luan and G. Yuan, Opt. Express, 2017, 25, 13648-13658.

26 A. E. Serebryannikov, E. Ozbay and S. Nojima, Opt. Express, 2014, 22, 3075-3088.

27 M. Stolarek, D. Yavorskiy, R. Kotynski, C. J. Zapata Rodriguez, J. Lusakowski and T. Szoplik, Opt. Lett., 2013, 38, 839-841.

28 Z. Li, S. Chen, C. Tang, W. Liu, H. Cheng, Z. Liu, J. Li, P. Yu, B. Xie, Z. Liu, J. Li and J. Tian, Appl. Phys. Lett., 2014, 105, 201103.

29 T. Aba, Y. Qu, T. Wang, Y. Chen, H. Li, Y. Wang, Y. Bai and Z. Zhang, Opt. Express, 2018, 26, 1199-1205.

30 C. Pan, M. Ren, Q. Li, S. Fan and J. Xu, Appl. Phys. Lett., 2014, 104, 121112.

31 D. F. Tang, C. Wang, W. K. Pan, M. H. Li and J. F. Dong, Opt. Express, 2017, 25, 11329-11339.

32 V. A. Fedotov, P. L. Mladyonov, S. L. Prosvirnin, A. V. Rogacheva, Y. Chen and N. I. Zheludev, Phys. Rev. Lett., 2006, 97, 167401.

33 L. Wu, Z. Yang, Y. Cheng, Z. Lu, P. Zhang, M. Zhao, R. Gong, X. Yuan, Y. Zheng and J. Duan, Opt. Express, 2013, 21, 52395246.

34 C. Menzel, C. Helgert, C. Rockstuhl, E. B. Kley, A. Tunnermann, T. Pertsch and F. Lederer, Phys. Rev. Lett., 2010, 104, 253902. 
35 M. Kim, K. Yao, G. Yoon, I. Kim, Y. Liu and J. Rho, Adv. Opt. Mater., 2017, 5, 1700600.

36 G. Yoon, I. Kim, S. So, J. Mun, M. Kim and J. Rho, Sci. Rep., 2017, 7, 6668.

37 Y. H. Wang, R. C. Jin, J. Li, J. Q. Li and Z. G. Dong, Opt. Express, 2018, 26, 3508-3517.

38 C. Menzel, C. Rockstuhl and F. Lederer, Phys. Rev. A, 2010, 82, 053811.

39 W.-Z. Xu, Y.-T. Shi, J. Ye, F.-F. Ren, I. V. Shadrivov, H. Lu, L. Liang, X. Hu, B. Jin, R. Zhang, Y. Zheng, H. H. Tan and C. Jagadish, Adv. Opt. Mater., 2017, 5, 1700108.
40 Z. Li, S. Chen, W. Liu, H. Cheng, Z. Liu, J. Li, P. Yu, B. Xie and J. Tian, Plasmonics, 2015, 10, 1703.

41 S. Liu, A. Noor, L. L. Du, L. Zhang, Q. Xu, K. Luan, T. Q. Wang, Z. Tian, W. X. Tang, J. G. Han, W. L. Zhang, X. Y. Zhou, Q. Cheng and T. J. Cui, ACS Photonics, 2016, 3, 1968-1977.

42 L. Zhang, R. Y. Wu, G. D. Bai, H. T. Wu, Q. Ma, X. Q. Chen and T. J. Cui, Adv. Funct. Mater., 2018, 28, 1802205. 